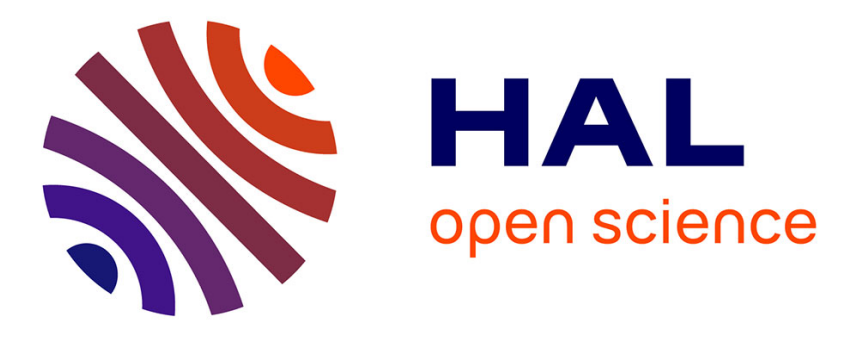

\title{
Optimization of beam parameters and iodine quantification in dual-energy contrast enhanced digital breast tomosynthesis
}

Sylvie Puong, Xavier Bouchevreau, Nicolas Duchateau, Razvan Iordache, Serge Müller

\section{To cite this version:}

Sylvie Puong, Xavier Bouchevreau, Nicolas Duchateau, Razvan Iordache, Serge Müller. Optimization of beam parameters and iodine quantification in dual-energy contrast enhanced digital breast tomosynthesis. SPIE Conference on Medical Imaging, 2008, San Diego, United States. pp.69130Z, 10.1117/12.770148 . hal-02320759

\section{HAL Id: hal-02320759 \\ https://hal.science/hal-02320759}

Submitted on 19 Oct 2019

HAL is a multi-disciplinary open access archive for the deposit and dissemination of scientific research documents, whether they are published or not. The documents may come from teaching and research institutions in France or abroad, or from public or private research centers.
L'archive ouverte pluridisciplinaire HAL, est destinée au dépôt et à la diffusion de documents scientifiques de niveau recherche, publiés ou non, émanant des établissements d'enseignement et de recherche français ou étrangers, des laboratoires publics ou privés. 


\title{
Optimization of beam parameters and iodine quantification in Dual-Energy Contrast Enhanced Digital Breast Tomosynthesis
}

\author{
Sylvie Puong ${ }^{a, b}$, Xavier Bouchevreau $^{a}$, Nicolas Duchateau ${ }^{c}$, Razvan Iordache $^{a}$ and Serge \\ Muller $^{a}$ \\ ${ }^{a}$ GE Healthcare, Buc, France; \\ ${ }^{b}$ Université Paris XI, Orsay, France; \\ 'Institut d'Optique Graduate School, Palaiseau, France
}

\begin{abstract}
Dual-Energy Contrast Enhanced Digital Breast Tomosynthesis (DE CEDBT) is a promising technique for breast cancer detection, which combines the strengths of functional and 3D imaging. In the present study, we first focused on the optimization of the acquisition parameters for the low and high-energy projections, which leads to a trade-off between image quality in the recombined slices and the Average Glandular Dose (AGD) delivered to the patient. Optimized parameters were found and experimentally validated on phantom images. Then, we addressed the problem of iodine quantification in the recombined slices. In DE CEDBT, iodine quantification is limited by the z-resolution, due to the restricted angle acquisition inherent to tomosynthesis. We evaluated the lesion thickness above which determination of iodine volumetric concentration is possible. For lesions below this thickness, estimation of iodine concentration is possible if a priori information or a model on the shape of the lesion is available. Iodine quantification for lesions located near the breast boundary is also challenging, due to scatter border effects and variation of the breast thickness in this region. A scatter correction algorithm based on a deconvolution scheme and a thickness compensation algorithm were applied on the low and high-energy projections. Corrected images showed a more accurate quantification of iodine.
\end{abstract}

Keywords: Digital X-ray, Mammography, Dual-Energy, Tomosynthesis, Scatter

\section{INTRODUCTION}

To travel distances over a millimeter, oxygen cannot be transported only via diffusion processes. This is the reason why organisms larger than a few millimeters need to develop a vascular system for oxygen transport to the organs, in order to grow and survive. The same phenomenon has been observed during cancer development, as angiogenesis occurs in the vicinity of tumours to support their growth. ${ }^{1}$ These newly formed blood vessels are characterized by an increased permeability, due to a lack of smooth muscle cells and pericytes in their walls, and cause the blood to pool around the tumors. Contrast Enhanced Digital Mammography (CEDM) takes advantage of this physiological phenomenon to enhance lesion visibility, by injecting an iodinated contrast medium into the blood. Combination of CEDM with tomosynthesis would further increase lesion conspicuity, with the production of image slices through the breast, minimizing therefore the problem of overlap encountered in projection imaging. ${ }^{2-4}$ Two approaches are possible to isolate the iodine contrast medium, which are the temporal subtraction and the dual-energy method, both resulting in the suppression of anatomical noise, while enhancing the visibility of contrast uptake. In this paper, we chose the dual-energy method, preferably to temporal subtraction, as it suffers less from patient motion artifacts, ${ }^{2}$ thanks to the quasi-simultaneous acquisition of the low and high-energy images which will be recombined to form the iodine-equivalent image. A few studies have reported the use of dual-energy in Contrast-Enhanced Digital Breast Tomosynthesis in the past. ${ }^{3-5}$ One aim of this paper was to determine the optimal low and high-energy acquisition parameters, in order to obtain a compromise between the image quality in the dual-energy recombined slices and patient dose. Image quality

Further author information:

S. Puong: E-mail: sylvie.puong@ge.com, Telephone: +33 (0)1 30704088 
was assessed in terms of lesion SDNR in the recombined slice. Optimization was done for a $5 \mathrm{~cm}$-thick, $50 \%$ glandular breast. We also investigated the problem of iodine quantification in DE CEDBT. Because of the limited z-resolution in tomosynthesis, only lesions with sufficient thickness have voxel values proportional to the iodine volumetric concentration in the recombined slices. ${ }^{4}$ Using simulated projections, we determined this threshold thickness for a given lesion size in the detector plane. For lesions under this threshold, a correction scheme was proposed to improve iodine quantification. Near the breast border, quantification is also disturbed by variable scatter behaviour and thickness changes. We studied the impact on iodine quantification of a scatter correction and a thickness compensation method.

\section{OPTIMIZATION OF BEAM PARAMETERS IN DE CEDBT}

In our DE CEDBT approach, at each angulation of the X-ray tube, a low-energy and a high-energy projection images are acquired successively in a very short time interval. Then, at each angulation, these projections are recombined into an iodine thickness-equivalent projection, using a specific dual-energy recombination algorithm. ${ }^{6}$ An iodine-equivalent volume is then reconstructed by the application of the Simultaneous Algebraic Reconstruction Technique ${ }^{7,8}$ (SART) on the iodine thickness-equivalent projections. In this part, we describe the optimization of the acquisition techniques, in order to reach a compromise between the image quality in the dual-energy recombined slices and patient dose.

\subsection{Method for the optimization of acquisition parameters in DE CEDBT}

Through an adequate choice of low and high-energy acquisition techniques, we sought to reach a trade-off between the image quality in the dual-energy recombined slice and patient dose. Image quality in the recombined slice was assessed by the Signal Difference to Noise Ratio (SDNR) of a given lesion. The worst-case scenario was considered by evaluating the visibility of the smallest lesion with the minimal concentration of iodine, i.e. when the total amount of iodine injected is assumed to have been uniformly diluted into the whole patient body volume. This corresponds to a $3 \mathrm{~mm}$-side cubic lesion with a volumetric concentration of iodine equal to 0.555 $\mathrm{mg} / \mathrm{cm}^{3}$.

The optimization was done by maximizing the SDNR in the recombined slice for a given target patient dose, and considering a $5 \mathrm{~cm}$-thick, $50 \%$ glandular breast. The target dose was chosen equal to twice the glandular dose for one standard mammogram, i.e. $2.4 \mathrm{mGy}$. In a previous article, ${ }^{4}$ we proved that the SDNR in the recombined slice is totally determined by the SDNR in the $0^{\circ}$ recombined projection. Therefore, it is equivalent to maximize the SDNR in the recombined slice for a total target patient dose of $2.4 \mathrm{mGy}$ and to maximize the SDNR in the $0^{\circ}$ recombined projection for a target dose equal to the total dose divided by the number of projections, i.e. $2.4 / 15=0.16 \mathrm{mGy}\left(15\right.$ projections between $-20^{\circ}$ and $\left.+20^{\circ}\right)$. Optimization of acquisition techniques for DE CEDBT is then equivalent to an optimization for Dual-Energy Contrast Enhanced Digital Mammography (DE CEDM). Derivation and simulation of the SDNR in the dual-energy recombined projection has been described in a previous article on DE CEDM. ${ }^{6}$

To further simplify the optimization process in DE CEDBT, we used the fact that the contrast of iodine in the $0^{\circ}$ recombined projection is always equal to the iodine thickness, independently of the low and highenergy acquisition techniques used, as a direct consequence of the dual-energy recombination algorithm design. ${ }^{6}$ Maximization of the SDNR in the $0^{\circ}$ recombined projection is then equivalent to minimizing the background noise in this recombined projection.

\subsection{Optimized acquisition parameters in DE CEDBT}

Using the method detailed in Sect. 2.1, a theoretical optimization of the acquisition parameters was performed, based on simulations of the SDNR in the $0^{\circ}$ recombined projection. In the present study, we restricted the optimization to anode/filter combinations developed for temporal subtraction CEDM, ${ }^{9}$ and which are also available on our prototype system based on a Senographe DS(GE Healthcare, Chalfont St Giles, UK): the anode and filter combinations considered were $\mathrm{Mo} / \mathrm{Mo}, \mathrm{Mo} / \mathrm{Rh}, \mathrm{Rh} / \mathrm{Rh}$ and $\mathrm{Mo} / \mathrm{Cu}$. For a $5 \mathrm{~cm}$-thick, $50 \%$ glandular breast and a target dose of $0.16 \mathrm{mGy}$, noise in the $0^{\circ}$ recombined projection was minimal when we used $\mathrm{Rh} / \mathrm{Rh}, 27 \mathrm{kVp}$, $90 \mathrm{mAs}$ (total mAs for the 15 projections) for low-energy and $\mathrm{Mo} / \mathrm{Cu}, 49 \mathrm{kVp}, 280 \mathrm{mAs}$ for high-energy. At this optimum, the ratio between the dose imparted to the low-energy projections to the total dose is equal to 0.48 . 


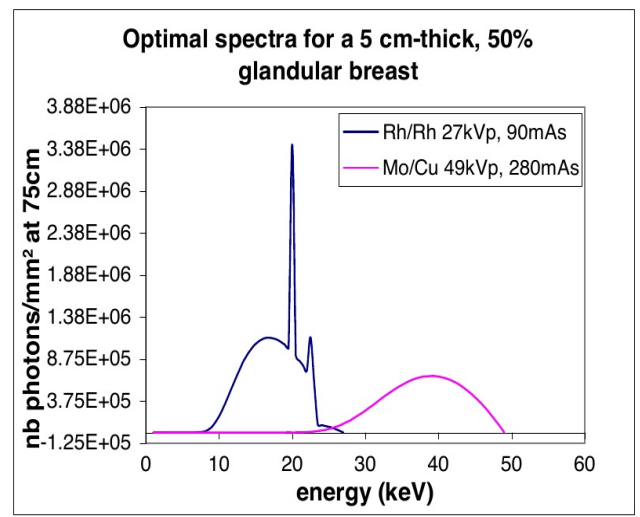

Figure 1. Optimal low and high-energy spectra for a $5 \mathrm{~cm}$-thick, $50 \%$ glandular breast: $\mathrm{Rh} / \mathrm{Rh}, 27 \mathrm{kVp}, 90 \mathrm{mAs}$ and $\mathrm{Mo} / \mathrm{Cu}, 49 \mathrm{kVp}, 280 \mathrm{mAs}$

Fig. 1 shows the shape of these optimal spectra. A preliminary study had been done, to find the optimal low and high energies and dose repartition between the low and high-energy images, with the same optimization method, in the ideal case of mono-energetic X-rays. Simulation of mono-energetic spectra led to optimal energies of 19 $\mathrm{keV}$ and $36 \mathrm{keV}$, with an optimal ratio of low-energy image dose to total dose of 0.51 . The mean energies of the poly-energetic spectra that we found by theoretical optimization, $17.5 \mathrm{keV}$ and $38 \mathrm{keV}$ for the low and the high-energy spectra respectively, with a low-energy dose ratio equal to 0.48 , are quite close to the ideal optimum found for mono-energetic spectra.

These results were validated with measurements on phantom images. We used a DSA phantom (Nuclear Associates - DSA phantom, linearity insert), which is composed of a $2.4 \mathrm{~cm}$ block of PMMA (material with attenuation equivalent to $50 \%$ glandular breast tissue) with embedded iodine inserts at different surfacic concentrations. Slabs of $50 \%$ breast-equivalent material (BR12 plates manufactured by CIRS) were added to reach a thickness of $5 \mathrm{~cm}$. The first aim of the experiments was to verify that the simulated background noise in the recombined projection was indeed close to the real value, measured on images. Another purpose was to validate the position of the optimum for the acquisition techniques. Three parameters had to be optimized: the shapes of the low-energy and high-energy spectra (determined by the anode/filter combination and the $\mathrm{kVp}$ ), and the ratio of the low-energy dose to the total dose. As it would have been difficult to show the optimum by simultaneously varying these three parameters, we chose to fix the low and high-energy spectra shapes, and vary the low-energy dose ratio in our experiments. The spectra shapes were chosen as the ones determined by simulations: Rh/Rh, $27 \mathrm{kVp}$ and $\mathrm{Mo} / \mathrm{Cu}, 49 \mathrm{kVp}$, for the low and high-energy respectively. Values of low and high-energy mAs were then chosen so as to have a constant total dose equal to $0.16 \mathrm{mGy}$ for the $0^{\circ}$ recombined projection, and low-energy dose ratios ranging from 0.4 to 0.9 . Corresponding acquisition parameters are shown in Table 1. The mAs values are the total $\mathrm{mAs}$ for the 15 projections.

Table 1. Acquisition parameters used in phantom experiments

\begin{tabular}{|l|c|c|c|c|c|c|}
\hline Low-energy dose ratio & 0.4 & 0.5 & 0.6 & 0.7 & 0.8 & 0.9 \\
\hline Total mAs for $\mathrm{Rh} / \mathrm{Rh}, 27 \mathrm{kVp}$ spectrum & 71 & 90 & 110 & 125 & 140 & 160 \\
\hline Total mAs for $\mathrm{Mo} / \mathrm{Cu}, 49 \mathrm{kVp}$ spectrum & 320 & 280 & 225 & 160 & 110 & 56 \\
\hline
\end{tabular}

Images of the DSA phantom were acquired with the above parameters, and the low and high-energy images were recombined using our dual-energy recombination algorithm. ${ }^{6}$ The background noise was measured in the recombined image by computing the standard deviation in a 100x100 pixels ROI located near the center of the phantom. These measurements were compared to the values simulated in our optimization algorithm. Fig. 2 shows a good agreement between simulated and experimental values of the background noise in the recombined projection, with an average relative error of $5 \%$. The variation of the noise with the low-energy dose ratio clearly 


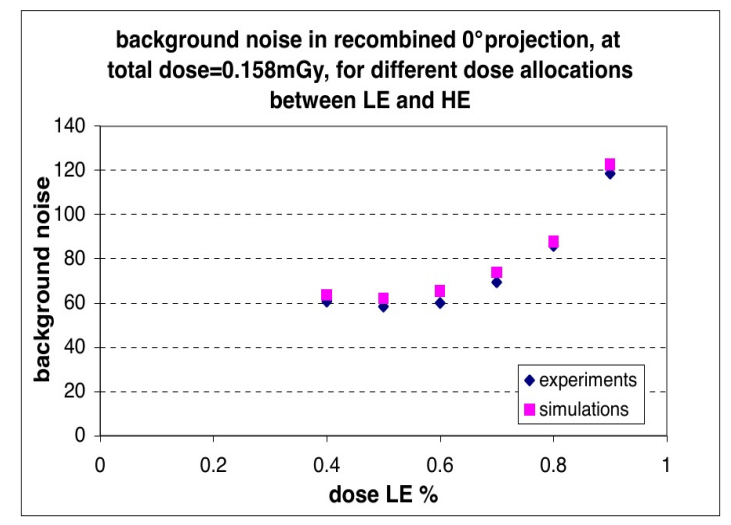

Figure 2. Variation of experimental and simulated values of background noise in the recombined projection with the low-energy dose ratio

displays an optimum at a low-energy dose ratio around 0.5 , on the experimental data, as expected from the simulations.

\section{IODINE QUANTIFICATION IN DE CEDBT}

Recent studies have suggested that tumor angiogenesis is associated with patient outcome in breast cancers. ${ }^{10}$ Microvessel density (MVD) calculation, which involves observation under a light microscope of immunohistochemically stained blood microvessels, is mainly used for quantification of tumor angiogenesis in breast cancer, and could be a prognostic factor in invasive breast cancer. Similarly, iodine amount in CEDBT could mirror the degree of vascularization of a tumor, and could help in determining the aggressiveness of the cancer. However, as discussed in our previous study, ${ }^{4}$ iodine quantification is not that straightforward in DE CEDBT, mainly due to the limited z-resolution in tomosynthesis, and to the presence of scattered radiation, but also to thickness variations near the breast border.

\subsection{Effect of thickness compensation near the breast border}

The dual-energy recombination algorithm used in our DE CEDBT approach is based on a model where the object thickness is constant. In the region where the breast is compressed, this hypothesis is verified, and we have, under the condition of minor perturbation from scattered radiation, a contrast value, in the recombined projection, proportional to the local iodine thickness. ${ }^{6}$ Nevertheless, near the breast border, where the thickness is no longer constant, the hypotheses of our model are no more satisfied, leading to errors in iodine quantification for lesions located in this region.

In order to compensate thickness variations, the dual-energy recombination algorithm first applies a thickness compensation scheme on the low-energy image, which corrects the gray levels in this region so that their values are in the same range as in the compressed breast region. This is done by fictitious addition of an adequate thickness of adipose tissue in the regions of varying thickness. Then, by determining a correspondence between the gray level values at low-energy and the gray level values at high-energy, for adipose tissue, we infer the adequate thickness of adipose tissue to add in the high-energy image in order to compensate the breast thickness variations. The compensated low and high-energy images are then recombined with the dual-energy recombination algorithm to produce the iodine-equivalent image.

We tried to evaluate the effect of thickness compensation on iodine quantification. In this purpose, we acquired low and high-energy images of an anthropomorphic phantom (CIRS stereotactic needle biopsy training phantom - Model 013), whose shape mimics that of a compressed breast. The anti-scatter grid was used in order to be able to distinguish the effects of scatter from the effects of varying thickness. In one experiment, blisters filled with iodine at two different concentrations (approximately $7 \mathrm{mg} / \mathrm{cm}^{2}$ and $10 \mathrm{mg} / \mathrm{cm}^{2}$ ) were placed in the middle of the phantom, where the thickness is constant, and in another experiment, in the border region, where the thickness varies. For each of these experiments, the low and high-energy projections were recombined with and without 


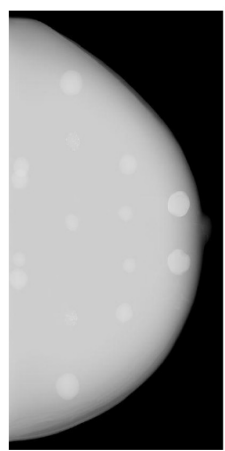

Fig. 3.a)

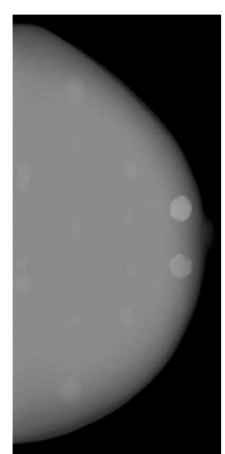

Fig. 3.b)

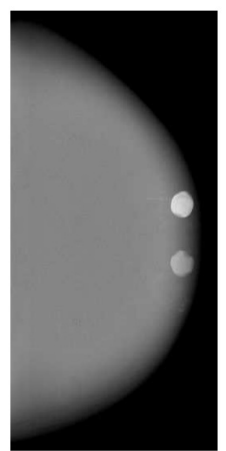

Fig. 3.c)

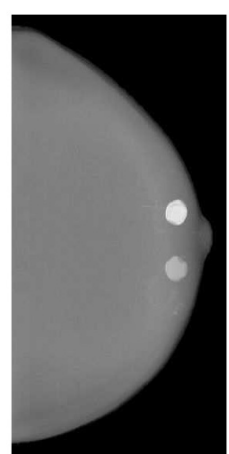

Fig. 3.d)

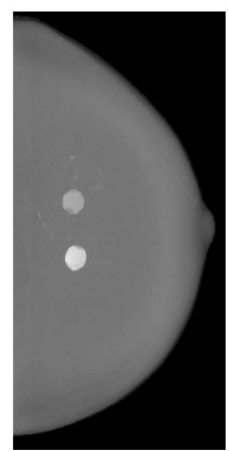

Fig. 3.e)

Figure 3. Recombined images of the anthropomorphic phantom:

a) Low-energy image ( $\mathrm{Rh} / \mathrm{Rh} 30 \mathrm{kVp}, 32 \mathrm{mAs})$. Two iodine blisters close to nipple, masses without iodine elsewhere

b) High-energy image (Mo/Cu $45 \mathrm{kVp}, 320 \mathrm{mAs}$ )

c) Recombined projection, without thickness compensation

d) Recombined projection, with thickness compensation

e) Recombined projection with iodine blisters in the center of the phantom, with thickness compensation

prior thickness compensation. When the iodine blisters are placed in the middle of the object, the conditions of the recombination model are satisfied. Therefore, we considered the iodine contrast in this configuration to be the correct one. The relative error on contrast, for the most concentrated iodine blister, compared to the contrast measured when the blister is placed in the phantom center, where the thickness is constant, is $0.07 \%$ with application of thickness compensation and $5.8 \%$ without compensation. Better visualization of tissues near the breast border was also achieved (see Fig. 3).

\subsection{Limited z-resolution impact on quantification accuracy}

Unlike computed tomography, where it is possible to acquire projections on an angular amplitude of $360^{\circ}$, the angular range is limited in tomosynthesis. For our experiments, the maximal angular amplitude was $40^{\circ}\left(-20^{\circ}\right.$ to $\left.+20^{\circ}\right)$.

In a previous article, ${ }^{4}$ we proved that the gray levels in the slices are proportional to the linear attenuation coefficient in the case of standard tomography, and to the iodine volumetric concentration in the case of dualenergy contrast enhanced tomography. But in the case of restricted angular range such as in tomosynthesis, the z-resolution severely decreases, and under a certain thickness of the lesion, the voxel value becomes proportional to the total iodine thickness in the lesion, rather than to the volumetric iodine concentration.

In this paper, we tried to determine the threshold value of lesion thickness above which the voxel value is proportional to the iodine volumetric concentration, so that iodine quantification is possible. In that purpose, 15 angulated projections between $-20^{\circ}$ and $+20^{\circ}$, of a lesion embedded in a $5 \mathrm{~cm}$-thick, $50 \%$ glandular breast were simulated. The lesion is a rectangular parallelepiped, with a square cross section in the detector plane. Proportionality of voxel values with the iodine volumetric concentration in dual-energy contrast enhanced tomography relies on the fact that voxel values are proportional to the linear attenuation coefficient in standard tomography. ${ }^{4}$ Therefore, the threshold value of lesion thickness above which iodine quantification is possible in DE CEDBT is the threshold above which we indeed have a voxel value proportional to the linear attenuation coefficient in standard tomosynthesis. Therefore, we reconstructed the simulated projections as in standard tomosynthesis, with the application of SART (3 iterations), without prior dual-energy recombination on the projections. The threshold thickness depends on the lesion size in the detector plane (xy plane), and the angular range of the projections. We determined this threshold for a $3 \times 3 \mathrm{~mm}$ lesion in the detector plane, for projections spanning $-20^{\circ}$ to $+20^{\circ}$. Lesions with different thicknesses (dimension along z-axis) were simulated, with a constant $3 \times 3$ $\mathrm{mm}$ size in the detector plane and a given linear attenuation coefficient, and the contrast of the lesion in the in-focus slice was measured for each of them. 


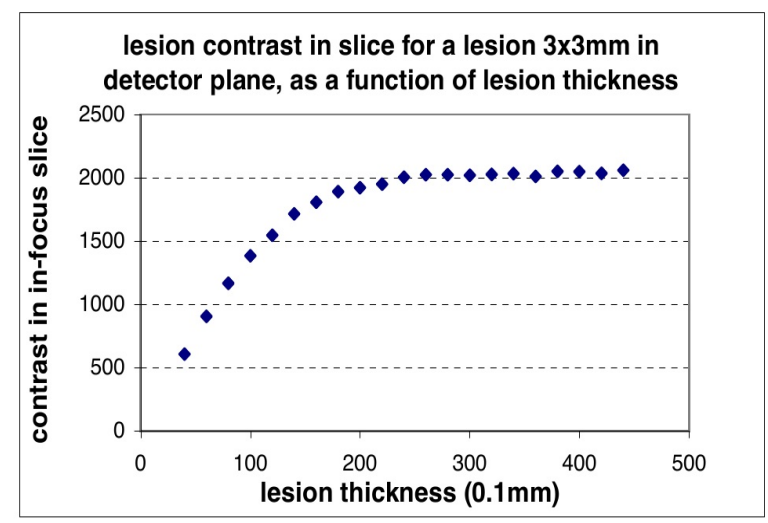

Fig. 4.a)

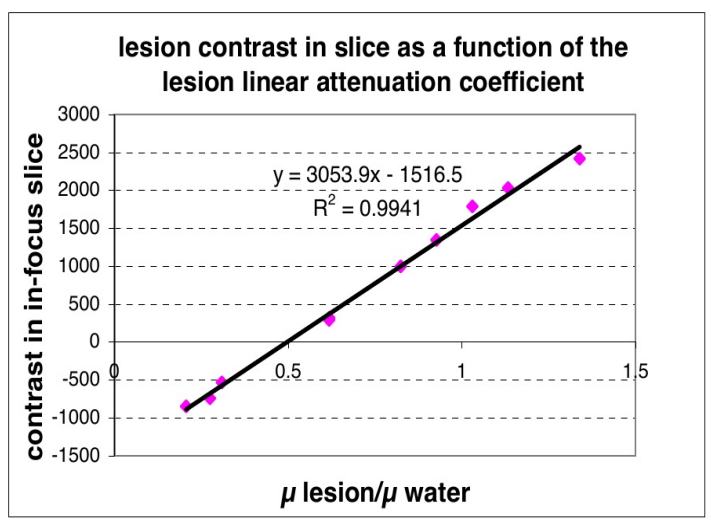

Fig. 4.b)

Figure 4. a) Variation of the contrast in the in-focus slice with the lesion thickness, for a lesion of $3 \mathrm{x} 3 \mathrm{~mm}$ in the detector plane, with a constant linear attenuation coefficient

b) Variation of the contrast in the in-focus slice with lesion linear attenuation coefficient, for a lesion of $3 \times 3 \mathrm{~mm}$ in the detector plane, with a thickness above $2 \mathrm{~cm}$

In Fig. 4.a), the contrast seems to vary linearly with the lesion thickness under a given threshold, and is constant above it. By analogy with the demonstration proposed in our previous article, ${ }^{4}$ under a certain threshold $T$, the contrast is proportional to $\mu \cdot t$, the product of the lesion linear attenuation coefficient by its thickness $t$ : contrast $=K \cdot \frac{\mu \cdot t}{T}$, with $K$ a constant. Above this threshold $T$, the contrast is proportional to the linear attenuation coefficient, which is constant here for all the simulated lesions: contrast $=K \cdot \mu$. To confirm the proportionality to the linear attenuation coefficient, we simulated lesions with a thickness above $2 \mathrm{~cm}$, which is greater than the threshold $T$ observed in Fig. 4.a), with $3 \times 3 \mathrm{~mm}$ size in the detector plane, as before, but with different linear attenuation coefficients. Figure 4.b) shows indeed a linearity between the lesion contrast in the in-focus slice and the lesion linear attenuation coefficient. Therefore, using the graph in Fig. 4.a), the threshold thickness $T$ above which iodine quantification is possible for a lesion of $3 \times 3 \mathrm{~mm}$ size in the detector plane can be determined as the transition thickness between the linearly varying and constant portions of the curve. Evaluation of the threshold $T$ was then done for lesions of different sizes $D$ in the detector plane. D corresponds to the side length of the lesion cross section in the detector plane, which is a square. As we plotted $T$ as function of $D$, we observed a linear relationship between the threshold thickness above which we can quantify iodine and the lesion size $D$ in the detector plane (Fig. 5). A linear regression on the values of $T$ and $D$ gave the following relationship:

$$
T=2.2 \cdot D+7.8 \mathrm{~mm}
$$

All these observations also hold for DE CEDBT, but with the iodine volumetric concentration instead of the linear attenuation coefficient.

Therefore, iodine quantification is only possible in extremely elongated lesions along the z-axis, which can be rarely encountered in realistic situations. Ideally, to correct the limited z-resolution effects on iodine contrast in the in-focus slice, this contrast should be multiplied by $T / t$, where $t$ is the lesion thickness. However, the lesion thickness is unknown. We approximated that the lesion thickness was equal to the lesion size $D$ in the detector plane, which is true in the case of spherical lesions. Then, we corrected the contrast in the in-focus recombined slice by multiplying it by $T / D$. In reality, for the majority of the lesions, the thickness is comprised between $D / 2$ and $2 D$. As a consequence, the corrected value of the contrast should lie somewhere between half the iodine volumetric concentration and twice the iodine volumetric concentration. In the hypothesis of a lesion thickness equally distributed between $D / 2$ and $2 D$, the average relative error in evaluating the iodine volumetric concentration is then $42 \%$, after correction by $T / D$. If no correction had been applied, i.e., if we had kept a contrast value equal to $\rho * t / T$, with $\rho$ the iodine volumetric concentration, this would have resulted in a larger relative error: if $D=3 \mathrm{~mm}$, then $T=14.5 \mathrm{~mm}$, so that $0.1 \cdot \rho \leq \rho \cdot t / T \leq 0.4 \cdot \rho$, which gives a mean relative error of $74 \%$. 


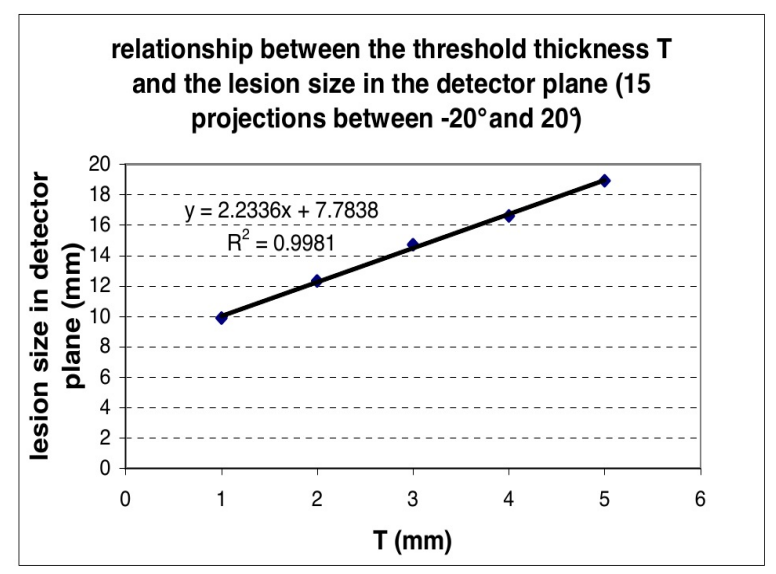

Figure 5. Threshold thickness $T$ as a function of lesion size in detector plane $D$

\subsection{Scattered radiation and iodine quantification}

The anti-scatter grid, used to remove secondary photons in standard mammography, has been designed for an $\mathrm{X}$-ray tube positioned at $0^{\circ}$. In tomosynthesis, this grid cannot be used any more for scatter removal, as the $\mathrm{X}$-ray tube assumes tilted positions with respect to the detector. When the tube is not at $0^{\circ}$, the lead septa would not be focused any more on the source position, leading to a severe cut-off of the primary beam.

Presently, tomosynthesis is performed without an anti-scatter grid, and a significant amount of scattered radiation is present in the projection images. Some papers have reported the subsequent effects of scatter in standard computed tomography (CT),${ }^{11,12}$ in standard tomosynthesis ${ }^{13}$ and in dual-energy CT. ${ }^{14}$ Among them, cupping artifacts, reconstruction values inaccuracy and decrease in the SDNR are the most invalidating.

We have previously shown some of these artifacts in DE CEDBT: ${ }^{4}$ the presence of the cupping artifact, and the error in iodine quantification in the dual-energy recombined projections, which led to an overestimation of the iodine amount for the lesions near the breast border, compared to the lesions located in the middle of the breast. In this paper, a first attempt was made at correcting scatter in DE CEDBT. Our approach was to remove scatter in the projections and, thus, restore the primary low and high-energy projection images. In a first step, we focussed on the projections at $0^{\circ}$, but further studies will address the problem of correcting scatter at angulated views.

The blurring effect of scatter on an image can be well modeled by the convolution of the primary image with a kernel. ${ }^{15}$ This kernel can be thought of as the scatter Point Spread Function (PSF), a map of the scattered photons impinging on the detector, and generated by a infinitely thin X-ray beam passing through a given scattering material. Correction of scatter can then be performed by iterative deconvolution schemes: ${ }^{16}$ a first estimate of the scatter image is produced by the convolution of the scatter PSF with the primary plus scatter image (the original image acquired), and then deducted from the primary plus scatter image to generate a first estimate of the primary; then, the estimation of the scatter is refined by convolving this first estimate of the primary with the scatter PSF, leading, after deduction from the scatter plus primary image, to a second estimate of the primary; the estimation of scatter is therefore refined by iterating this process several times.

Here, the scatter PSF was determined by a theoretical calculation, which has been detailed in Appendix A. We assessed our scatter correction method on images of the DSA phantom, with added slabs of PMMA, such that the total thickness was $6 \mathrm{~cm}$. Images were acquired without the anti-scatter grid, at $0^{\circ}$, with acquisition parameters typical of those used in DE CEDBT. Here, we used the optimal parameters determined in Sect. 2.2: $\mathrm{Rh} / \mathrm{Rh}, 27 \mathrm{kVp}, 6.3 \mathrm{mAs}$ for low-energy and $\mathrm{Mo} / \mathrm{Cu}, 49 \mathrm{kVp}, 18 \mathrm{mAs}$, for high-energy. As we can observe in Fig. 6, the cupping artifact has been significantly reduced in the dual-energy recombined image. This is also shown by the profiles in Fig. 7. Quantification of iodine in the recombined image has also been improved by the scatter correction: the linearity of the variations of iodine contrast in the recombined image with the iodine thickness is more respected after correction, as the overestimation of iodine thickness in the iodine inserts located 

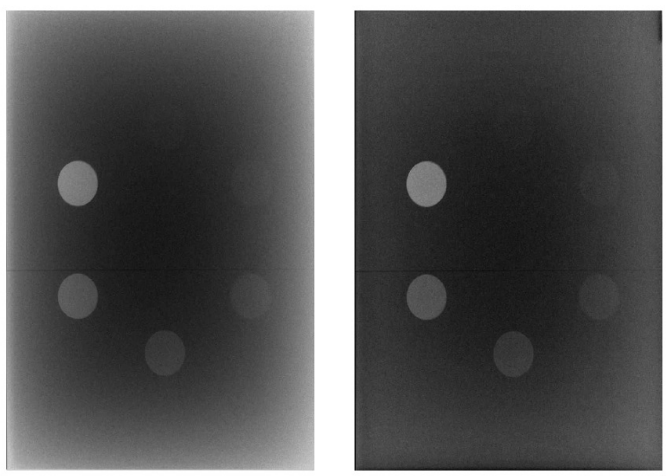

Figure 6. Dual-energy recombined images of the DSA phantom (6 cm-thick), with (left) and without (right) scatter correction
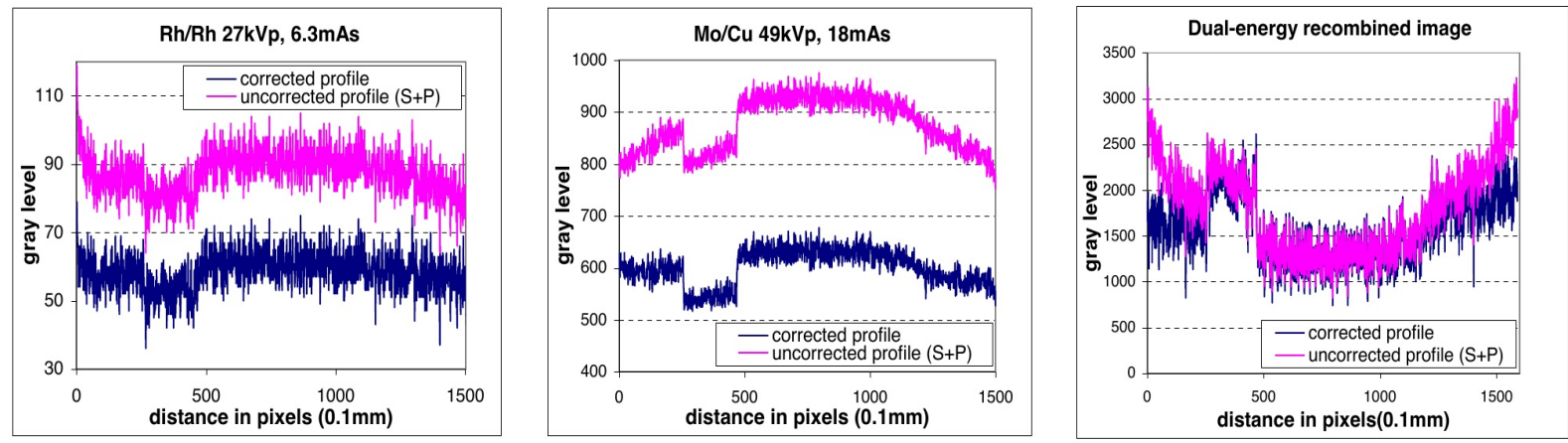

Figure 7. Profiles through an iodine insert, with and without scatter correction, in the low-energy, high-energy and recombined images of the DSA phantom $(6 \mathrm{~cm}$-thick)

near the border, farther from the chest wall (anode heel effect also plays a role in the overestimation), has been partly compensated by the correction (see Fig. 8). Linear regression on the contrast values with respect to iodine thickness shows indeed a higher correlation coefficient $\left(R^{2}=0.99\right)$ after scatter correction, than without scatter correction $\left(R^{2}=0.97\right)$.

However, the cupping artifact is not totally suppressed with this scatter correction method, nor the iodine thickness value for quantification totally restored. Indeed, limitations of our approach include neglecting higher order scatter interactions, which leads to an underestimation of the scatter PSF. In fact, it has been proved
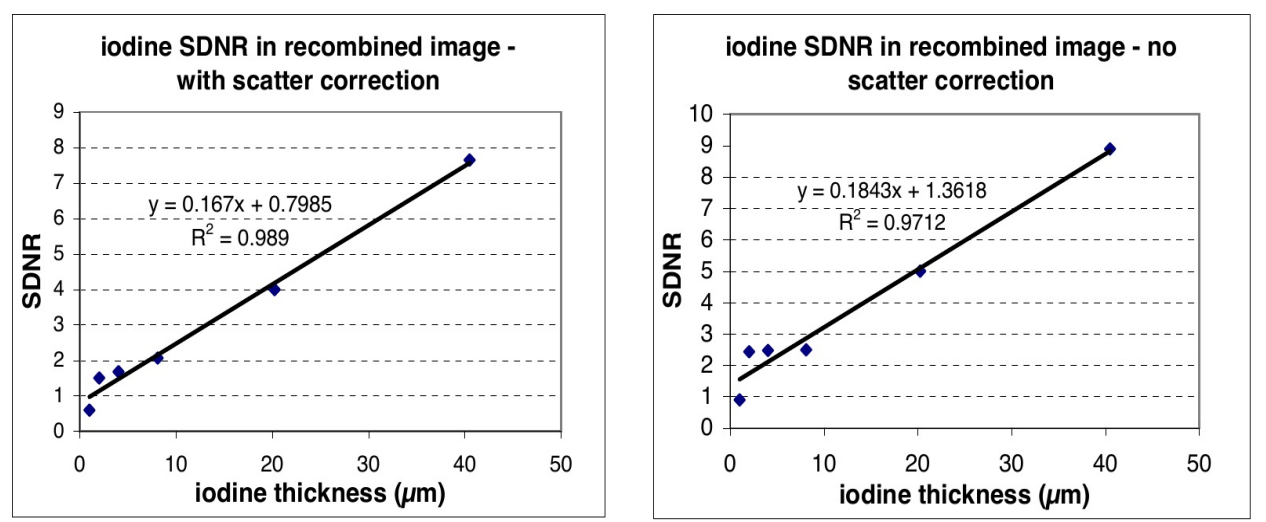

Figure 8. Iodine contrast in the recombined image of DSA phantom (6 cm-thick), with and without scatter correction, as a function of the iodine thickness. Inserts with 2 and $4 \mu \mathrm{m}$ iodine thickness are the nearest from the phantom border, and at the opposite side of the chest wall (on the right in the images in Fig. 6) 
that scatter interactions of second and above orders account for nearly half of the total scatter for thicknesses between 5 and $10 \mathrm{~cm} .{ }^{17}$ In addition, in the present correction algorithm, the object is assumed to be of uniform composition, and the local thickness is deduced from the gray level at each pixel. Practically, the composition and the thickness both vary across the breast, and their values cannot be inferred directly from the gray level. A refinement to our method could consider the breast to be divided into two regions: the region of compressed breast, where the thickness is constant and the composition varies; and the breast border region, where the composition is assumed constant (mainly fatty tissues) and the thickness is allowed to vary. Therefore, in each of these regions, the thickness or composition can be deduced from the gray level value.

\section{CONCLUSION}

This paper is a further development of the DE CEDBT approach introduced in a previous study, ${ }^{4}$ in which we provide the optimal acquisition parameters to be used with this new application. These were determined in order to reach a compromise between image quality in the recombined slices and patient dose. Optimization by consideration of the SDNR and dose for the recombined slices is equivalent to optimization by consideration of the SDNR and dose for the $0^{\circ}$ recombined projection, so that the approach developed in our study on DE $\mathrm{CEDM}^{6}$ is applicable. The problem of angle limitation in tomosynthesis and its influence on slice contrast and iodine quantification in DE CEDBT has also been investigated. We determined the lesion thickness above which iodine quantification is directly possible from contrast measurement in the in-focus recombined slice. A method to correct for the effect of poor z-resolution is introduced, under the assumption of lesions with a spherical shape. Iodine quantification has also been ameliorated near the breast border, by the application of a thickness compensation algorithm and a scatter correction on the low and high-energy projections. Further studies should investigate the correction of scatter in angulated views, as well as the inclusion of higher order scatter interactions in the PSF.

\section{APPENDIX A. THEORETICAL DERIVATION OF THE SCATTER PSF}

Because of the complexity of scatter interactions of higher orders, only first order scatter will be taken into account in this calculation of the scatter PSF. We consider an infinitely thin pencil beam of X-rays, normally incident on the detector. As we suppose a scattering medium infinitely extended along the $\mathrm{x}$ and $\mathrm{y}$-axis (the xy plane is the plane of the detector), we can assume an isotropic behaviour of scatter in all directions, so that the scatter PSF has an axial symmetry around the z-axis. Calculation of the PSF can therefore be done in the xz plane, or any plane containing the z-axis, the resulting profile being revolved around the z-axis to obtain the whole scatter PSF. The following formula was used to derive the scatter profile in the xz plane:

$$
N(r)=\rho \int_{E=E_{\min }}^{E_{\max }} \int_{l=0}^{d} \frac{d \sigma}{d \Omega}(E, l, r) \cdot \mathrm{d} \Omega(l, r) \cdot I_{\text {element }}(E, l) \cdot e^{-\mu(E) l_{2}} \mathrm{~d} l \mathrm{~d} E
$$

where $\rho$ is the scattering medium density,

$\frac{d \sigma}{d \Omega}(E, l, r)$ is the scatter differential cross section, derived from the data from Hubbell, ${ }^{18}$

$d \Omega=\frac{d S \cdot \cos \theta}{l_{1}^{2}}$ is the solid angle from which is seen the element $d S$ of the detector, from the scattering element, $I_{\text {element }}(E, l)$ is the number of photons of energy $E$, reaching the scattering element at depth $l: I_{\text {element }}(E, l)=$ $I_{0} \cdot e^{-\mu(E) l}$, with $I_{0}(E)$, the number of photons of energy $E$ incident on the object.

The scatter PSF profile $K(r)$ is then obtained by normalizing $N(r)$ by the value of the primary radiation:

$$
K(r)=\frac{N(r)}{\int_{E=E_{\min }}^{E_{\max }} I_{0}(E) \cdot e^{-\mu(E) d} \mathrm{~d} E}
$$

The X-ray beam is polychromatic, and its spectrum $I_{0}(E)$ is calculated with an algorithm simulating the image formation chain. 


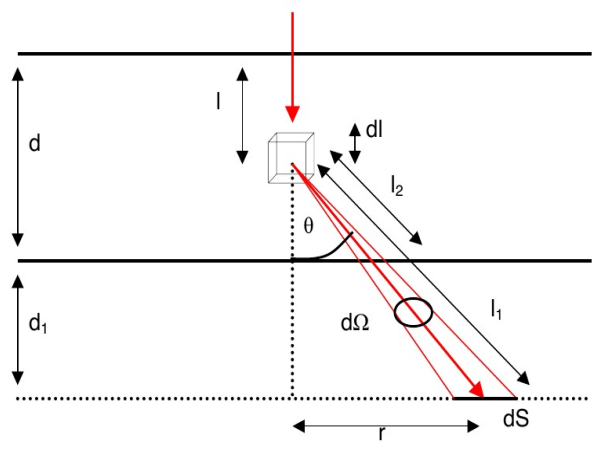

Figure 9. Theoretical derivation of scatter PSF: the pixel at distance $r$ from the PSF centre receives the energy scattered by the element of thickness $d l$

This document is the property of GE Healthcare and shall be kept confidential under the SPIE rule: "Privileged information or ideas obtained through access to conference abstracts and manuscripts prior to presentation or publication for the purpose of conference organization or peer review must be kept confidential and must not be used for competitive gain"

\section{ACKNOWLEDGMENTS}

Funding for this study has been supplied by the French Ministry of Research, under the grant ANR05RNTS00101, and by the ANRT, under the CIFRE convention 2005/496.

This document is the property of GE Healthcare and shall be kept confidential under the SPIE rule: "Privileged information or ideas obtained through access to conference abstracts and manuscripts prior to presentation or publication for the purpose of conference organization or peer review must be kept confidential and must not be used for competitive gain"

\section{REFERENCES}

[1] Frouge, C., Guinebretière, J., Paola, R. D., Contesso, G., and Blery, M., "Correlation between contrast enhancement and tumor angiogenesis," Investigative Radiology 29, 1043-1049, (1994).

[2] Carton, A.-K., Li, J., Albert, M., Chen, S., and Maidment, A., "Quantification for contrast-enhanced digital breast tomosynthesis," in Medical Imaging 2006: Physics of Medical Imaging, Flynn, M. and Hsieh, J., eds., Proc. SPIE 6142, 111-121, (2006).

[3] Carton, A.-K., Lindman, K., Ullberg, C., Francke, T., and Maidment, A., "Dual-energy subtraction for contrast-enhanced digital breast tomosynthesis," in Medical Image 200\%: Physics of Medical Imaging, Flynn, M. and Hsieh, J., eds., Proc. SPIE 6510, 651007, (2007).

[4] Puong, S., Patoureaux, F., Iordache, R., Bouchevreau, X., and Muller, S., "Dual-energy contrast enhanced digital breast tomosynthesis: concept, method, and evaluation on phantoms," in Medical Image 200\%: Physics of Medical Imaging, Flynn, M. and Hsieh, J., eds., Proc. SPIE 6510, 65100U, (2007).

[5] Glick, S. J. and Didier, C., "A computer simulation for evaluating dual-energy, contrast-enhanced breast tomosynthesis," in Medical Image 200\%: Physics of Medical Imaging, Flynn, M. and Hsieh, J., eds., Proc. SPIE 6510, 65102V, (2007).

[6] Puong, S., Bouchevreau, X., Patoureaux, F., Iordache, R., and Muller, S., "Dual-energy contrast enhanced digital mammography using a new approach for breast tissue canceling," in Medical Image 2007: Physics of Medical Imaging, Flynn, M. and Hsieh, J., eds., Proc. SPIE 6510, 65102H, (2007).

[7] Andersen, A. and Kak, A., "Simultaneous Algebraic Reconstruction Technique (SART): a superior implementation of the ART algorithm," Ultrasonic Imaging 6, 81-94, (1984).

[8] Zhang, Y., Chan, H.-P., Sahiner, B., Wei, J., Goodsitt, M., Hadjiiski, L., Ge, J., and Zhou, C., "A comparative study of limited-angle cone-beam reconstruction methods for breast tomosynthesis," Medical Physics 33(10), 3781-3795, (2006). 
[9] Skarpathiotakis, M., Yaffe, M., Bloomquist, A., Rico, D., Muller, S., Rick, A., and Jeunehomme, F., "Development of contrast digital mammography," Medical Physics 29(10), 2419-2426, (2002).

[10] Gasparini, G., "Clinical significance of determination of surrogate markers of angiogenesis in breast cancer," Critical Reviews in Oncology and Hematology 37, 97-114, (2001).

[11] Joseph, P. M. and Spital, R. D., "The effects of scatter in x-ray computed tomography," Medical Physics 9(4), 464-472, (1982).

[12] Siewerdsen, J. H. and Jaffray, D. A., "Cone-beam computed tomography with a flat-panel imager: magnitude and effects of scatter," Medical Physics 28(2), 220-231, (2001).

[13] Wu, G., Mainprize, J. G., Boone, J. M., and Yaffe, M. J., "Evaluation of scatter effects on image quality for breast tomosynthesis," in Medical Image 2007: Physics of Medical Imaging, Flynn, M. and Hsieh, J., eds., Proc. SPIE 6510, 65101T, (2007).

[14] Vetter, J. R. and Holden, J. E., "Correction for scattered radiation and other background signals in dualenergy computed tomography material thickness measurements," Medical Physics 15(5), 726-731, (1988).

[15] Seibert, J. and Boone, J., "X-ray scatter removal by deconvolution," Medical Physics 15(4), 567-575, (1988).

[16] Trotter, D. G., Tkaczyk, J., Kaufhold, J., Claus, B., and Eberhard, J., "Thickness-dependent scatter correction algorithm for digital mammography," in Medical Image 2002: Physics of Medical Imaging, Antonuk, L. and Yaffe, M., eds., Proc. SPIE 4682, 469-478, (2002).

[17] Boone, J. M. and Seibert, J. A., "Monte carlo simulation of the scattered radiation distribution in diagnostic radiology," Medical Physics 15(5), 713-720, (1988).

[18] Hubbell, J. H. and Øverbø, I., "Relativistic atomic form factors and photon coherent scattering cross sections," Journal of Physical and Chemical Reference Data 8(1), 69-106, (1979). 